\title{
Wide band simultaneous multi-frequency single pulse study of PSR J1822-2256 with upgraded GMRT
}

\author{
Bhal Chandra Joshi ${ }^{1}$, Arun Naidu ${ }^{2}$, Vishal Gajjar ${ }^{3}$ \\ and Geoffrey A. E. Wright ${ }^{4}$ \\ ${ }^{1}$ National Centre for Radio Astrophysics (TIFR), Postbag No 3, Ganeshkhind, Pune INDIA \\ email: bcj@ncra.tifr.res.in \\ ${ }^{2}$ Mcgill Space Institute, McGill University, Montreal Canada \\ ${ }^{3}$ Space Science Laboratory, University of California, Berkeley USA \\ ${ }^{4}$ Jodrell Bank Centre for Astrophysics, University of Manchester UK
}

\begin{abstract}
We present simultaneous multi-frequency observations of PSR J1822-2256 for the first time, utilizing the unique capabilities of upgraded Giant Meterwave Radio Telescope (uGMRT). No emission is detected in about $10 \%$ of pulses. At least two drift modes and a possibly third rare mode, occur for 66, 21 and $2 \%$ pulses respectively $\left(\mathrm{P}_{3} \sim 17,7.5\right.$ and $5 \mathrm{P}_{0}$ respectively). The three drift modes and the nulls occur concurrently from 250 to $1500 \mathrm{MHz}$. Modal average profiles are distinct with their widths increasing with drift rate. These sub-pulse drift related profile mode-changes can provide independent probes of beam geometry and polar gap physics.
\end{abstract}

Keywords. pulsars: individual : PSR J1822-2256, radiation mechanisms: general

\section{Introduction}

While the sub-pulse drift is believed to be related to polar gap potential (Leeuwen \& Timokhin 2012), relativistic plasma outflow appears to be diminished during pulse nulls, if OFF duration of intermittent pulsars is considered as an extreme form of nulling (Gajjar et al. 2012; Lyne et al. 2010; Timokhin 2010). Both these phenomena occur concurrently over a wide band of frequencies (Gajjar et al. 2014; Naidu et al. 2017a). In some pulsars, changes in sub-pulse drift organizes the average emission in distinct profile modes (Wright \& Fowler 1981; Vivekanand \& Joshi 1997). Unlike sub-pulse drift $\left(\mathrm{P}_{3}\right)$ and nulls, these profile modes are dependent on the observing frequency and the beam geometry. Thus, deep simultaneous multi-frequency studies of pulsars with distinct drift rates can provide independent probes of geometry and polar gap physics. PSR J1822-2256 is a $1.8 \mathrm{sec}$ pulsar, with a single component profile at 610 and $1400 \mathrm{MHz}$, developing a leading component at $325 \mathrm{MHz}$. Three distinct drift rates and pulse nulling were reported at $610 \mathrm{MHz}$ (Naidu et al. 2017a). Here, we present recent observations of this interesting pulsar for the first time using the new unique capabilities of upgraded Giant Meterwave Radio Telescope (uGMRT).

\section{Simultaneous multi-frequency observations with uGMRT}

GMRT has recently been upgraded with installation of wide-band feeds covering 250-500 MHz, 550-950 MHz and 1100-1500 MHz (Band 3, 4 and 5 respectively) and $400 \mathrm{MHz}$ wide-band digital backend providing 4 phased array beams (Gupta et al. 2017). 
These upgrades make uGMRT a unique instrument providing a seamless frequency coverage from $250-1500 \mathrm{MHz}$ with high sensitivity, making it an ideal instrument for our study. PSR J1822-2256 was observed in July 2017 using the uGMRT in a multi-beam mode, employing Band 3, 4 and 5 with $400 \mathrm{MHz}$ bandwidth. The pulse sequences, aligned using time-stamps derived from an atomic clock, were obtained after folding dedispersed data to 512 bins across the period using TEMPO2 predictors (See Gajjar et al. 2014 for details of analysis).

We present for the first time a detailed study of single pulse behaviour of PSR J1822-2256 using the unique wide-band capabilities of uGMRT. PSR J1822-2256 shows prominent nulls, occurring concurrently from 250 to $1500 \mathrm{MHz}$ with a nulling fraction of $10.1 \pm 1.4 \%$. Three drift modes were identified, using both visual examination and short time fluctuation spectra (STFS), with $\mathrm{P}_{3}$ of 17,7 and $5 \mathrm{P}_{0}$ (Mode $\mathrm{A}, \mathrm{B}$ and $\mathrm{C}$ respectively), occurring for 66,21 and $2 \%$ of pulses respectively. The drift mode switches simultaneously across all observing bands. Mode $\mathrm{C}$ is very rare and represents chaotic occurrence of sub-pulses. Average profiles for each mode, formed from mode separated pulses using STFS analysis are distinctly different with a leading component becoming more prominent for Mode B as compared to Mode A. The width of Mode B profile is also larger than Mode A. The presence of multiple profile modes, associated with changes in sub-pulse drift, reported here for the first time, makes this pulsar as the sixth such pulsar, similar to PSRs B0031-07, J1727-2739, B1918+19, B2303+30, B2319+60 (Joshi 2013; Rankin et al. 2013; Wen et al. 2016).

\section{Discussions}

The multiple drift modes in PSR J1822-2256 can be interpreted in a manner similar to the mode-changes of PSR B1918+19, which was modeled as a carousel of 12 "sparks" circulating at a fixed speed, where the modes are observed as first-order aliases with each mode change corresponding to a loss of a single spark (Rankin et al. 2013). Applying this model to PSR J1822-2256, we assume 12 sparks initially observed under aliasing to drift at $\mathrm{P}_{3} \sim 17 \mathrm{P}_{0}$ with a constant circulation time of $12 \times 17 / 16 \mathrm{P}_{0}=12.8 \mathrm{P}_{0}$, not radically different from the value predicted by Ruderman \& Sutherland (1975)'s original model $\left(5.2 \mathrm{P}_{0}\right)$. Then, when one spark is lost its $\mathrm{P}_{3}$ changes to $17 \times 12 /(17+11)=7.3 \mathrm{P}_{0}$, as observed. We note also that the sub-pulse spacing increases (i.e. the profile width) as would be expected by this model. Finally, a further loss of a spark predicts a $\mathrm{P}_{3}$ of 4.6 $\mathrm{P}_{0}$ for Mode $\mathrm{C}$, close to the estimated value.

\section{References}

Gajjar, V., Joshi, B. C., \& Kramer, M. 2012, MNRAS, 424, 1197

Gajjar, V., Joshi, B. C., Kramer, M., Karuppusamy, R., \& Smits, R. 2014, ApJ, 797,18

Gupta, Y. et al. 2017, Current Science, 113, 707

Joshi, B. C. 2013, Proc. IAU Symp., 291, 414

van Leeuwen, J. \& Timokhin, A. N. 2012, ApJ, 752, 155

Lyne, A., Hobbs, G., Kramer, M., Stairs, I., \& Stappers, B. 2010, Science, 329, 408

Naidu, A., Joshi, B. C., Manoharan, P. K., \& Krishnakumar, M. 2017, A $\& A$ A 604, A45

Rankin., J., Wright, G. \& Brown, A. 2013, MNRAS, 433, 445

Ruderman, M. A. \& Sutherland, P. G. 1975, ApJ, 196, 51

Timokhin, A. N. 2010, MNRAS, 408, L41

Vivekanand, M. \& Joshi, B. C. 1997, ApJ, 477, 431

Wen, Z. G. et al. 2016, A\&A 592, A127

Wright, G. A. \& Fowler, L. A. 1981, A\&\&A, 101, 356 